\title{
The association of three DNA repair genes polymorphisms on the frequency of chromosomal alterations detected by fluorescence in situ hybridization
}

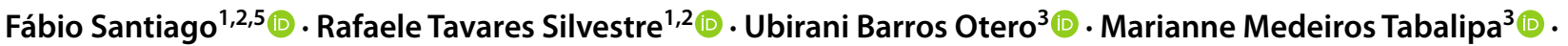 \\ Marilza de Moura Ribeiro-Carvalho ${ }^{1,2}$ (D) - Luciano Rios Scherrer ${ }^{4}$ (D) Ahmed Al-Rikabi ${ }^{5}$ (D) Thomas Liehr $^{5}$ (D) . \\ Gilda Alves $^{1,2}$ (I) Maria Helena Ornellas ${ }^{1,2} \mathbb{D}$
}

Received: 19 June 2020 / Accepted: 7 January 2021 / Published online: 28 March 2021

(C) The Author(s) 2021, corrected publication 2021

\begin{abstract}
Purpose Gas station workers (GSWs) are exposed to carcinogenic agents. The aim was to study the association of high somatic chromosome alterations (CAs) rates in the blood of GSWs and the polymorphisms of three genes playing a role in DNA double-strand break repair.

Methods This is a cross-sectional study with $114 \mathrm{GSWs}$ and 115 age-matched controls. Cytogenetic analyses, blood exams, medical interviews and genotypes for RAD51/G135C (rs1801320), ATM/P1054R (rs1800057) and CHEK2/T470C (rs 17879961) genes were performed.

Results The CA rate in GSWs was 9.8 CAs/1000 metaphases, and $19.1 \%$ of the workers had $>10$ CAs per 1000 metaphases (group two). GSWs had decreased levels of monocytes $(P=0.024)$ in their blood exams. The number of variant alleles of the $R A D 51 / G 135 C$ polymorphism was higher in GSWs $(P=0.011)$ compared to the controls, and were associated with enhanced number of CAs per worker $(P=0.008)$. No allele variant was found for $C H E K 2 / T 470 C$ in this study.

Conclusion The RAD51/G135C polymorphism appears to be related to genome instability in gas station workers. Increasing the knowledge of DNA repair gene variations involved in maintaining genomic stability in GSWs may be crucial for future cancer prevention.
\end{abstract}

Keywords Benzene $\cdot$ Gas station worker $\cdot R A D 51 / G 135 C \cdot A T M / P 1054 R \cdot$ Chromosome aberration

Gilda Alves

galvesbrown@gmail.com

1 Laboratory of Circulating Biomarkers, Department of Pathology, Faculty of Medical Sciences, Rio de Janeiro State University (UERJ), Avenida Professor Manuel de Abreu 444, $4^{\circ}$ andar, Vila Isabel, Rio de Janeiro 20551-030, Brazil

2 Graduation Program of Medical Sciences (PGCM), Medical Sciences Faculty (FCM), Rio de Janeiro State University, Rio de Janeiro, Brazil

3 Technical Area Environmental, Work and Cancer, Coordination of Prevention and Surveillance, National Cancer Institute José Alencar Gomes da Silva (INCA), Rio de Janeiro, Brazil

4 Kennedy Faculties of Belo Horizonte, Minas Gerais, Brazil

5 Jena University Hospital, Friedrich Schiller University, Institute of Human Genetics, Jena, Germany

\section{Introduction}

BTEX (benzene, toluene, ethyl benzene, and xylene) are aromatic hydrocarbons widely used as solvents in fuels, being chemical contaminants in gas stations' environments. Benzene is considered the main carcinogenic agent (group one according to IARC), and the association with cancer is well-established (IARC 2018; Falzone et al. 2016). In Brazil, as self-service fuel filling at gas stations is illegal, gas station workers (GSWs) have to fill the fuel in the car's tank; due to this, they are chronically exposed to high concentrations of BTEX. Although the mechanisms by which BTEX cause genotoxic effects are not fully clear, there is evidence that the function of a wide range of cellular targets are perturbed by specific metabolites and reactive oxygen species (ROS). Genotoxic effects include: (one) inhibition of topoisomerase II; (two) adduct formation of reactive metabolites; (three) oxidative DNA 
damage; (four) error-prone DNA repair; and (five) epigenetic alterations (McHale et al. 2011; Dewi et al. 2020).

Chromosome alterations (CAs) are standardized biomarkers of early biological effects in human biomonitoring. In fact, CAs in peripheral blood lymphocytes reflect inter-individual sensitivity to exogenous genotoxic substances and can be used as biomarker of carcinogenic risk (Rossner et al. 2005; Mateuca et al. 2012; Zhang et al. 2012; Li et al. 2015; Villalba-Campos et al. 2016). Fluorescence in situ hybridization (FISH) using whole chromosome painting (wcp) probes allows a rapid detection of CAs, enabling new possibilities of cytogenetic dosimetry (Verdorfer et al. 2001; Santiago et al. 2014).

Lower activity of the DNA repair mechanisms may generate higher somatic rates of CAs, favoring the development of cancer (De Palma and Manno 2014). The DNA repair genes $R A D 51, A T M$, and $C H E K$ play a role in the DNA double-strand break repair preventing CAs; however, some polymorphisms could made this task less efficient.

The aims of this study were to assess the frequency of the RAD51/G135C (rs1801320), ATM/P1054R (rs 1800057) and CHEK2/T470C (rs 17879961) polymorphisms and their putative association with the CAs, along with the evaluation of the health of 114 GSWs in Rio de Janeiro. The polymorphisms RAD51/G135C, ATM/ $P 1054 R$, and CHEK2/T470C were selected because they were associated with many cancers, such as prostate, breast, head and neck cancer, and leukemias (Skasko et al. 2009; Schumacher et al. 2018; Zeng et al. 2018).

\section{Subjects and methods}

\section{Population study}

This is a cross-sectional study with 114 workers (60 men and 54 women) recruited at 11 gas stations in Rio de Janeiro and 115 age-matched controls (64 men and 51 women). A trained interviewer questioned the members of the study population regarding their age, sex, skin color (self-declaration), life-style (smoking habits, alcohol and illicit drug consumption, etc.) and about their offspring (Table 1). The control groups were recruited among administrative workers, cleaning workers, and nurses (not exposed to chemotherapy neither X-rays) of two hospitals, in a church (housewives and workers) and teaches. By the questionnaire, we did not detected high exposure to tobacco, alcohol consumption or industrialized food intake. Individuals showing alterations in the blood test were excluded from the control group. The minimum period of exposure for the GSW was 6 months. If the participant had undergone any kind of surgery, either was exposed to X-ray, or was infected by arboviruses in 3 months

Table 1 Demographics data of gas station workers

\begin{tabular}{|c|c|c|c|c|c|c|}
\hline Data & Group 1 & Group 2 & $\begin{array}{l}\text { Group } \\
1 \times \text { Group } \\
2 \\
(P \text {-value })\end{array}$ & Total of workers & Controls & $\begin{array}{l}\text { Total of workers } \times \\
\text { controls }(P \text { value })\end{array}$ \\
\hline Gender & & & 0.035 & & & 0.235 \\
\hline Women & $39(42.4 \%)$ & $15(68.2 \%)$ & & $54(47.4 \%)$ & $64(55.7 \%)$ & \\
\hline Men & $53(57.6 \%)$ & $7(31.8 \%)$ & & $60(52.6 \%)$ & $51(44.3 \%)$ & \\
\hline Age (year) & $38.9( \pm 12.4)$ & $38.8( \pm 12.51)$ & 0.900 & $38.84( \pm 12.42)$ & $36.43( \pm 12.93)$ & 0.101 \\
\hline Time of employment (year) & 5.7. $( \pm 6.0)$ & $4.3( \pm 3.8)$ & 0.574 & & & \\
\hline Skin color & & & 0.638 & & & 0.100 \\
\hline Black & $25(27.2 \%)$ & $8(36.4 \%)$ & & $33(28.9 \%)$ & $20(18.2 \%)$ & \\
\hline White & $14(15.7 \%)$ & $3(13.6 \%)$ & & $17(14.9 \%)$ & $48(43.6 \%)$ & \\
\hline Brown (Mulatto) & $50(54.3 \%)$ & $10(45.5 \%)$ & & $60(52.6 \%)$ & $41(37.3 \%)$ & \\
\hline Light brown (Native Indians) & $2(2.2 \%)$ & $0(0.0 \%)$ & & $2(1.8 \%)$ & $1(0.9 \%)$ & \\
\hline Yellow (Asiatic) & $1(1.1 \%)$ & $1(4.5 \%)$ & & $2(1.8 \%)$ & $0(0.0 \%)$ & \\
\hline \multicolumn{7}{|l|}{ Alcohol consumption } \\
\hline No & & & & $18(15.8 \%)$ & $52(45.2 \%)$ & 1.000 \\
\hline Yes & & & & $75(65.8 \%)$ & $51(44.3 \%)$ & \\
\hline Stopped drinking & & & & $15(13.2 \%)$ & $5(4.3 \%)$ & \\
\hline Never drank & & & & $6(5.3 \%)$ & $7(6.1 \%)$ & \\
\hline First trimester of spontaneous abortion & & & & $7(77.8 \%)$ & $1(11.1 \%)$ & 0.015 \\
\hline
\end{tabular}

$P$ value $<0.05$ was considered significant

Descriptive measures: $a( \pm b), a=$ average and $b=$ standard deviation 
before the interview, man or woman was excluded from the study. No medication causing CA was reported by the participants. No test for virus was conducted in the blood of the subjects, nevertheless we asked for previous diseases. The subjects reported no hereditary condition although some have reported cases of cancer in the family. It was unclear if the cancer was hereditary. No significant difference was found in smoking cigarettes, alcohol consumption or industrialized food intake between the GSW group and the control's.

Peripheral blood samples were collected for complete hemogram, biochemistry and cytogenetic tests. The workers were divided into two groups (group one, $\leq 10$ chromosomal abnormalities per 1,000 metaphases; and group two, $>10$ chromosomal abnormalities per 1000 metaphases) and compared to clinical characteristics and genotyping results.

\section{Cytogenetic analyses}

The cytogenetic analyses were performed for delimiting GSWs at risk as previously described and for allowing associations between the frequency of lymphocyte CAs, genotyping results, and clinical characteristics (Zhang et al. 2012; Verdorfer et al. 2001; Santiago et al. 2014). Blood samples, $2 \mathrm{~mL}$ of heparinized whole blood, were collected by venipuncture. Lymphocyte cultures were performed and chromosomes were prepared according to standard procedures after $48 \mathrm{~h}$ of cultivation (Liehr and Claussen 2002). FISH was done as previously reported using homemade wcp probes for chromosomes one, two, and four (Verdorfer et al. 2001; Santiago et al. 2014). One-hundred metaphases were analyzed per GSW and 200 metaphases in 11/115 controls.

\section{Genotyping}

Genomic DNA from peripheral blood leukocytes was obtained by phenol-chloroform extraction and analyzed by polymerase chain reaction and restriction enzyme digestion (PCR-RFLP) assays for RAD51/G135C (rs1801320), $A T M / P 1054 R$ (also known as $3161 \mathrm{C}>\mathrm{G}$, rs 1800057), and CHEK2/T470C (rs17879961) polymorphisms according to previous publications (Skasko et al. 2009; Green and Sambrook 2012; Schumacher et al. 2018). The PCR reactions were carried out in the VeritiVR Thermal Cycler (Applied Biosystems) and were done using 50-200 ng of genomic DNA, $0.4 \mu \mathrm{M}$ of each primer, $1 \times$ PCR buffer, $250 \mu \mathrm{M}$ of dNTPs, $1.5 \mathrm{mM}$ of $\mathrm{MgCl}_{2}$, and 1-2.5 units of Taq polymerase in a $50 \mu \mathrm{L}$ reaction volume. PCR products were digested with MvaI (RAD51/G135C, $60{ }^{\circ} \mathrm{C}$ for $\left.1 \mathrm{~h}\right)$, AlwI (ATM/P1054R, $37^{\circ} \mathrm{C}$ for $1 \mathrm{~h}$ ), and PstI (CHEK2/T430C, $37^{\circ} \mathrm{C}$ for $5 \mathrm{~min}$ ) (New England Biolabs), and then separated by electrophoresis in $10 \%$ polyacrylamide, and the digested/ separated products were further visualized by silver staining.
Positive and negative controls were used in all reactions. Note that for $R A D 51 / G 135 C$ polymorphism, the wild allele is represented by the letter "G" (Guanine) and the variant allele by "C" (Cytosine). While for ATM/P1054R polymorphism, the wild allele is represented by the letter "C" (Cytosine) and the variant allele by "G" (Guanine).

\section{Statistical analysis}

The Hardy-Weinberg (HW) equilibrium was tested using the Chi-Square $\left(\chi^{2}\right)$ statistic for the goodness-of-fit test for each polymorphism, and the differences in the allele and genotype frequencies between groups were analyzed using standard $\chi^{2}$ or Fisher's exact tests. In the distributed variables, a nonparametric Mann-Whitney test or Goodness-of-fit test (multinomial distribution) was used for comparison of the distributed variables between groups using the IBM SPSS (version 2.0). The odds ratio (OR) was also calculated. For all statistical tests, $P$ value $<0.05$ was considered significant.

\section{Results}

\section{Clinical and demographic data}

The GSWs interviewed in this study routinely worked for six days a week, for eight hours or more per day, with 6.9 years of median time of employment. Regarding age, there were no significant differences between the workers $(38.84 \pm 12.42)$ and the control groups $(36.43 \pm 12.93)(P=0.101)$. As for skin color (self-declaration), $52.6 \%$ (60/115) self-declared as brown (Mulatto), 14.9\% (17/115) white, 28.9\% (33/115) black, $1.8 \%$ (2/115) light brown (as Native Indians), and $1.8 \%(2 / 115)$ yellow (as Asiatic). A low prevalence of smoking (7\%) and moderate consumption of alcohol beverage were identified. No statistical differences were found between alcohol consumption, illicit drug use (marijuana, cocaine, and ecstasy), smoking, and race between workers and controls $(P=1.000 ; P=1.000 ; P=0.293 ; P=0.100$, respectively; see Table 1). Despite no statistical difference for gender between workers and controls, a higher number of women were observed in group two (68.2\%) compared to group one $(42.4 \%)(P=0.035)$.

Regarding the comparative analyses of blood tests, monocytes, eosinophils, basophils, hemoglobin (men), hematocrit (men), and gamma-gt were found to be significantly higher in the workers group, when compared with the controls $(P=0.001 ; P<0.001 ; P<0.001, P=0.001, P=0.003$, and $P<0.001$, respectively). On the other hand, platelets, erythrocytes (women), hemoglobin (women), and neutrophil levels $(P=0.001, P=0.001, P=0.003$ and $P=0.001$, respectively) were lower. It should be noted that only monocytes 
Table 2 Laboratory data of gas station workers

\begin{tabular}{|c|c|c|c|c|c|c|}
\hline Data & Group 1 & Group 2 & $\begin{array}{l}\text { Group } \\
1 \times \text { Group } \\
2 \\
(P \text { value })\end{array}$ & Total of workers & Controls & $\begin{array}{l}\text { Total of workers } \times \\
\text { controls }(P \text { value })\end{array}$ \\
\hline Platelets $\left(10^{9} / \mathrm{L}\right)$ & $254.16( \pm 58.83)$ & $255.52( \pm 70.85)$ & 0.969 & $254.43( \pm 60.99)$ & $278.29( \pm 49.70)$ & 0.001 \\
\hline Gamma-GT (U/L) & $37.17( \pm 39.40)$ & $28.81( \pm 12.61)$ & 0.819 & $35.74( \pm 35.86)$ & $27.00( \pm 37.30)$ & $<0.001$ \\
\hline Direct bilirubin (mg/dL) & $0.39( \pm 0.17)$ & $0.34( \pm 0.15)$ & 0.223 & $0.14( \pm 0.05)$ & $0.16( \pm 0.07)$ & 0.046 \\
\hline Leukocytes $(/ \mu \mathrm{L})$ & $7318.1( \pm 2102.3)$ & $7226.2( \pm 1291.2)$ & 0.795 & $7300.22( \pm 1966.01)$ & $7778.61( \pm 1980.38)$ & 0.066 \\
\hline Neutrophils (\%) & $55.41( \pm 9.80)$ & $55.78( \pm 8.36)$ & 1.00 & $55.49( \pm 9.51)$ & $59.46( \pm 10.92)$ & 0.001 \\
\hline Eosinophils (\%) & $3.10( \pm 2.76)$ & $2.73( \pm 1.61)$ & 0.951 & $3.03( \pm 2.58)$ & $2.14( \pm 3.09)$ & $<0.001$ \\
\hline Basophils (\%) & $0.42( \pm 0.34)$ & $0.33( \pm 0.23)$ & 0.577 & $0.40( \pm 0.32)$ & $0.27( \pm 0.43)$ & $<0.001$ \\
\hline Typical lymphocytes (\%) & $33.62( \pm 8.92)$ & $35.05( \pm 7.89)$ & 0.385 & $33.89( \pm 8.72)$ & $\pm 32.44( \pm 9.79)$ & 0.152 \\
\hline Monocytes (\%) & $7.40( \pm 2.04)$ & $6.24( \pm 1.55)$ & 0.024 & $7.18( \pm 2.00)$ & $5.42( \pm 1.95)$ & $<0.001$ \\
\hline Reticulocytes (\%) & $\pm 1.13( \pm 0.38)$ & $1.19( \pm 0.30)$ & 0.209 & $1.15( \pm 0.36)$ & $1.27( \pm 0.46)$ & 0.080 \\
\hline \multicolumn{7}{|l|}{ Women } \\
\hline $\begin{array}{l}\text { Erythrocytes (million/ } \\
\quad \mu \mathrm{L})\end{array}$ & $4.48( \pm 0.22)$ & $4.31( \pm 0.35)$ & 0.148 & $4.44( \pm 0.37)$ & $4.62( \pm 0.48)$ & 0.056 \\
\hline Hemoglobin & $13.06( \pm 1.1)$ & $12.54( \pm 1.09)$ & 0.117 & $12.92( \pm 1.11)$ & $13.58( \pm 1.88)$ & 0.021 \\
\hline Hematocrit (\%) & $38.76( \pm 2.98)$ & $37.42( \pm 3.02)$ & 0.216 & $38.40( \pm 3.02)$ & $40.18( \pm 3.56)$ & 0.002 \\
\hline $\begin{array}{l}\text { Mean corpuscular vol- } \\
\text { ume (fl) }\end{array}$ & $86.66( \pm 5.21)$ & $86.86( \pm 3.73)$ & 0.845 & $86.72( \pm 4.82)$ & $87.12( \pm 5.13)$ & 0.511 \\
\hline \multicolumn{7}{|l|}{ Men } \\
\hline $\begin{array}{l}\text { Erythrocytes (million/ } \\
\mu \mathrm{L} \text { ) }\end{array}$ & $4.98( \pm 0.37)$ & $4.95( \pm 0.11)$ & 0.904 & $4.98( \pm 0.35)$ & $4.72( \pm 0.45)$ & 0.001 \\
\hline Hemoglobin & $14.47( \pm 1.13)$ & $14.49( \pm 0.71)$ & 0.884 & $14.47( \pm 1.08)$ & $13.82( \pm 2.08)$ & 0.003 \\
\hline Hematocrit (\%) & $42.37( \pm 2.86)$ & $42.51( \pm 1.55)$ & 1.000 & $42.38( \pm 2.72)$ & $42.23( \pm 3.71)$ & 0.370 \\
\hline $\begin{array}{l}\text { Mean corpuscular vol- } \\
\text { ume (fl) }\end{array}$ & $84.90( \pm 4.89)$ & $84.90( \pm 2.42)$ & 0.645 & $85.42( \pm 4.35)$ & $88.07( \pm 5.07)$ & 0.001 \\
\hline
\end{tabular}

$P$ value $<0.05$ was considered significant

Normal values: Platelets $150-40010^{9} / \mathrm{L}$; gamma-GT $8-71 \mathrm{U} / \mathrm{L}$; direct bilirubin up to $0.3 \mathrm{mg} / \mathrm{dL}$; leukocytes $4000-10,000 / \mu \mathrm{L}$; neutrophils 40-75\%; eosinophils 1-6\%; basophils 0-1\%; typical lymphocytes $20-45 \%$; monocytes $2-10 \%$, reticulocytes $0.5-2 \%$; erythrocytes $4.5-6.5$ million $/ \mu \mathrm{L}$; hemoglobin $13.5-18 \mathrm{~g} / \mathrm{dL}$, hematocrit 40-54\%, mean corpuscular volume 76-96 fl. Descriptive measures: $a( \pm b)$, $a=$ average and $b=$ standard deviation

were associated with a high CA rate $(P=0.024$, group one vs. group two), as showed in Table 2.

\section{The CA data}

The GSWs CA rate was 9.8 CAs/1000 metaphases, and a high frequency of CAs (>10 CAs per 1000 metaphases) was found in $19.1 \%(22 / 114)$ of GSWs, whereas $80.9 \%$ (92/114) of workers showed no aberrations or less than ten CAs per 1000 metaphases, and no CAs were found among controls.

Chromosome one with $38.4 \%$ (43/112) of CAs was the most affected, followed by chromosomes four (32.1\%) and two $(29.4 \%)$; however, no statistical difference was found between the chromosomes and CA distribution $(P=0.494)$. Among the total CAs, the translocations were most frequently found (38.4\%), followed by monosomies $(14.3 \%)$;

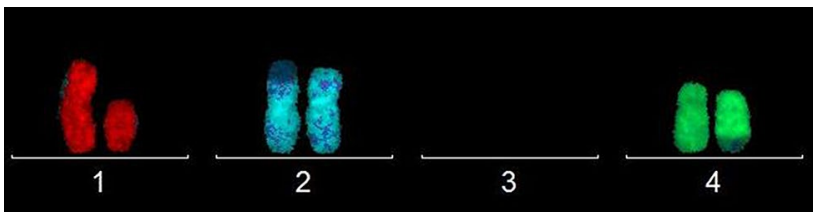

Fig. 1 CAs found in analyses of one female worker. CAs$\operatorname{del}(1), \operatorname{der}(2), \mathrm{t}(2 ; ?), \operatorname{der}(4), \mathrm{t}(4 ; ?)$. The homemade probes were conjugated with TexasRed to label chromosome 1 (red), Diethylaminocoumarin (DEAC) for chromosome 2 (lightblue), and fluorescein isothiocyanate (FITC) for chromosome 4 (green). Other chromosomes were counterstained with DAPI (dark blue)

deletions (13.4\%); chromosomal fragments (13.4\%); chromosomal breaks (11.6\%); chromosome derivatives $(5.3 \%)$; trisomies (1.8\%), and inversion (1.8\%). Figure 1 shows an example of CAs found in one female worker (CAs$\operatorname{del}(1), \operatorname{der}(2), \mathrm{t}(2 ; ?), \operatorname{der}(4), \mathrm{t}(4 ; ?))$. 


\section{Genotyping}

The RAD51/G135C, ATM/P1054R, and CHEK2/T470C polymorphisms were determined for GSWs and controls. The RAD51/G135C and ATM/P1054R polymorphisms did not show deviation from the HW equilibrium in the population analyzed ( $P=0.322, P=0.632$, respectively), as shown in Table 3. However, the variant genotype (TC and CC) CHEK2/T430C was not found in GSWs or controls; thus, these results were not considered for statistical analysis. Neither $R A D 51 / G 135 C$ nor $A T M / P 1054 R$ polymorphisms were associated with gender or ethnicity. In a comparative population analysis, the frequencies of ATM/P1054R showed no statistical difference between

Table 3 Genotypic frequencies of RAD51/G135C and ATM/P1054R genotypes in 114 gas station workers and 115 controls

\begin{tabular}{|c|c|c|c|c|c|c|}
\hline & \multicolumn{3}{|l|}{$R A D 51 / G 135 C$} & \multicolumn{3}{|l|}{ ATM/P1054R } \\
\hline & GG & $\mathrm{GC}$ & $\mathrm{CC}$ & $\mathrm{CC}$ & $\mathrm{GC}$ & GG \\
\hline Group 1 & $59(64.1 \%)$ & $29(31.5 \%)$ & $4(4.3 \%)$ & $70(79.5 \%)$ & $18(20.5 \%)$ & $0(0.0 \%)$ \\
\hline Group 2 & $9(40.9 \%)$ & $10(45.5 \%)$ & $3(13.6 \%)$ & $19(86.4 \%)$ & $2(9.1 \%)$ & $1(4.5 \%)$ \\
\hline Total of workers $N(\%)$ & $68(59.6 \%)$. & $38(33.3 \%)$ & $8(7.0 \%)$ & $89(80.9 \%)$ & $20(18.2 \%)$ & $1(0.9 \%)$ \\
\hline Controls $N(\%)$ & $88(72.2 \%)$ & $24-(21.1 \%)$ & $2(1.8 \%)$ & $92(82.1 \%)$ & $20(17.9 \%)$ & $0(0.0 \%)$ \\
\hline \multirow[t]{2}{*}{ Total } & $156(68.4 \%)$ & $63(27.6 \%)$ & $9(3.9 \%)$ & $181(81.5 \%)$ & $40(18.0 \%)$ & $1(0.4 \%)$ \\
\hline & \multicolumn{3}{|l|}{$P$ value } & \multicolumn{3}{|l|}{$P$ value } \\
\hline Hardy-Weinberg Equilibrium & \multicolumn{3}{|l|}{0.322} & \multicolumn{3}{|l|}{0.632} \\
\hline Total workers $\times$ controls & \multicolumn{3}{|l|}{0.011} & \multicolumn{3}{|l|}{0.930} \\
\hline Group $1 \times$ Group 2 & \multicolumn{3}{|l|}{0.074} & \multicolumn{3}{|l|}{0.092} \\
\hline \multicolumn{7}{|c|}{ Polymorphisms assessment for CA detection } \\
\hline & \multicolumn{2}{|l|}{$R A D 51 / G 135 C$} & \multicolumn{2}{|l|}{ ATM/P1054R } & \multicolumn{2}{|c|}{$R A D 51 / G 135 C+A T M / P 1054 R$} \\
\hline Specificity (\%) & \multicolumn{2}{|l|}{$87(76-94)$} & $79(68-87)$ & \multicolumn{3}{|c|}{$82(75-88)$} \\
\hline Sensitivity (\%) & \multicolumn{2}{|l|}{$28(16-43)$} & $14(16-43)$ & \multicolumn{3}{|c|}{$24(14-35)$} \\
\hline
\end{tabular}

$P$ value $<0.05$ was considered significant

Descriptive measures: $a( \pm b), a=$ average and $b=$ standard deviation. $(a-b)$, confidence interval sample, $95 \%$

Table 4 Associations between genotypic frequencies of $R A D 51 / G 135 C$ in 114 GSW and biometrics (cytogenetic and demographic) data

\begin{tabular}{|c|c|c|c|c|c|c|}
\hline & \multicolumn{3}{|l|}{$R A D 51 / G 135 C$} & \multirow{2}{*}{$\begin{array}{l}P \text { value } \\
\mathrm{GG} \times \mathrm{GC}\end{array}$} & \multirow{2}{*}{$\begin{array}{l}P \text { value } \\
\mathrm{GG} \times \mathrm{CC}\end{array}$} & \multirow{2}{*}{$\begin{array}{l}P \text { value } \\
\mathrm{GC}+\mathrm{CC} \times \mathrm{GG}\end{array}$} \\
\hline & GG & GC & $\mathrm{CC}$ & & & \\
\hline \multicolumn{7}{|l|}{ Gender $N(\%)$} \\
\hline Men & $35(51.5 \%)$ & $20(52.6 \%)$ & $6(75.0 \%)$ & 1.000 & 0.275 & 0.702 \\
\hline \multicolumn{7}{|l|}{ Ethnicity $N(\%)$} \\
\hline Women & $33(48.5 \%)$ & $18(47.4 \%)$ & $2(25.0 \%)$ & & & \\
\hline Black & $18(26.5 \%)$ & $12(31.6 \%)$ & $3(37.5 \%)$ & 0.555 & 0.164 & 0.395 \\
\hline Mulatto & $40(58.8 \%)$ & $18(47.4 \%)$ & $3(37.5 \%)$ & & & \\
\hline White & $9(13.2 \%)$ & $6(15.8 \%)$ & $1(12.5 \%)$ & & & \\
\hline Asiatic & $0(0.0 \%)$ & $1(2.6 \%)$ & $1(12.5 \%)$ & & & \\
\hline Native Indians & $1(1.5 \%)$ & $1(1.5 \%)$ & $0(0.0 \%)$ & & & \\
\hline $\begin{array}{l}\text { Number of } \\
\text { abnormal } \\
\text { metaphases } \\
\text { per subject }\end{array}$ & $0.59( \pm 1.4)$ & $0.76( \pm 1.00)$ & $1.63( \pm 1.6)$ & 0.028 & 0.004 & 0.005 \\
\hline $\begin{array}{l}\text { Number of } \\
\text { chromosomal } \\
\text { aberrations per } \\
\text { subject }\end{array}$ & $1.63( \pm 1.92)$ & $0.84( \pm 1.94)$ & $1.03( \pm 1.33)$ & 0.034 & 0.011 & 0.008 \\
\hline
\end{tabular}


total workers and controls $(P=0.930)$; however, by the Chi-Square test, the frequencies of RAD51/G135C were different $(P=0.011)$ (see Table 3$)$, indicating higher frequency of the $R A D 51 / G 135 C$ variant in the GSW population.

There was a positive association for a number of CAs per GSW and variants of $R A D 51 / G 135 C$ genotypes $(P=0.008$, $\mathrm{GG}+\mathrm{GC} \times \mathrm{CC} ; P=0.011 ; \mathrm{GG} \times \mathrm{CC}$; and $P=0.034$, GC $\times$ GG), as shown in Table 4. Similar results were found for the distribution of the number of abnormal metaphases per workers $(P=0.005, \mathrm{GG} \times \mathrm{GC}+\mathrm{CC} ; P=0.004 ; \mathrm{GG} \times \mathrm{CC}$; and $P=0.028, \mathrm{GG} \times \mathrm{GC}$ ) (see Table 4 ).

Regarding the comparative analyses for types of chromosomal alterations and $R A D 51 / G 135 \mathrm{C}$ genotypes, we found a higher number of chromosome fragments $(P=0.004, \mathrm{GG}$ $\times \mathrm{GC} ; P=0.014 ; \mathrm{GG} \times \mathrm{GC}+\mathrm{CC})$ and chromosome breaks $(P=0.013, \mathrm{GG} \times \mathrm{GC})$ between variant allele genotype groups (Table 5).

The frequencies of $A T M / P 1054 R$ genotypes were compared between the workers and controls, and no significant difference was detected, indicating that the two
Table 5 Associations between genotypic frequencies of $R A D 51 / G 135 C$ and types of chromosome alterations

\begin{tabular}{|c|c|c|c|c|c|c|}
\hline & \multicolumn{3}{|c|}{$R A D 51 / G 135 C$} & \multirow{2}{*}{$\begin{array}{l}P \text { value } \\
\mathrm{GG} \times \mathrm{GC}\end{array}$} & \multirow{2}{*}{$\begin{array}{l}P \text { value } \\
\mathrm{GG} \times \mathrm{CC}\end{array}$} & \multirow{2}{*}{$\begin{array}{l}P \text { value } \\
\mathrm{GG} \times \mathrm{CC}+\mathrm{GC}\end{array}$} \\
\hline & GG & GC & $\mathrm{CC}$ & & & \\
\hline \multicolumn{7}{|c|}{ Translocations } \\
\hline 0 & $58(85.3 \%)$ & $26(68.4 \%)$ & $6(75.0 \%)$ & 0.133 & 0.769 & 0.126 \\
\hline 1 & $6(8.8 \%)$ & $7(18.4 \%)$ & $1(12.5 \%)$ & & & \\
\hline 2 & $3(4.4 \%)$ & $4(10.5 \%)$ & $1(12.5 \%)$ & & & \\
\hline 4 & $0(0.0 \%)$ & $1(2.6 \%)$ & $0(0.0 \%)$ & & & \\
\hline 5 & $1(1.5 \%)$ & $0(0 \%)$ & $0(0.0 \%)$ & & & \\
\hline \multicolumn{7}{|c|}{ Chr. fragments } \\
\hline 0 & $64(94.0 \%)$ & $30(78.9 \%)$ & $8(100.0 \%)$ & 0.004 & 1.000 & 0.014 \\
\hline 1 & $2(2.9 \%)$ & $8(21.1 \%)$ & $0(0.0 \%)$ & & & \\
\hline 2 & $1(1.5 \%)$ & $0(0 \%)$ & $0(0.0 \%)$ & & & \\
\hline 3 & $1(1.5 \%)$ & $0(0 \%)$ & $0(0.0 \%)$ & & & \\
\hline \multicolumn{7}{|c|}{ Chr. Breaks } \\
\hline 0 & $63(94.0 \%)$ & $35(92.1 \%)$ & $6(75.0 \%)$ & 0.787 & 0.013 & 0.245 \\
\hline 1 & $3(4.5 \%)$ & $3(7.9 \%)$ & $0(0.0 \%)$ & & & \\
\hline 2 & $0(0.0 \%)$ & $0(0.0 \%)$ & $2(25.0 \%)$ & & & \\
\hline 3 & $1(1.5 \%)$ & $0(0.0 \%)$ & $0(0.0 \%)$ & & & \\
\hline \multicolumn{7}{|c|}{ Deletions } \\
\hline 0 & $62(91.2 \%)$ & $34(91.9 \%)$ & $6(75.0 \%)$ & 0.731 & 0.197 & 0.774 \\
\hline 1 & $4(5.9 \%)$ & $3(8.1 \%)$ & $1(12.5 \%)$ & & & \\
\hline 2 & $2(2.9 \%)$ & $0(0.0 \%)$ & $1(12.5 \%)$ & & & \\
\hline \multicolumn{7}{|c|}{ Chr. Derivatives } \\
\hline 0 & $65(95.6 \%)$ & $37(97.4 \%)$ & $8(100.0 \%)$ & 0.785 & 1.000 & 0.764 \\
\hline 1 & $1(1.5 \%)$ & $1(2.6 \%)$ & $0(0.0 \%)$ & & & \\
\hline 2 & $2(2.9 \%)$ & $0(0.0 \%)$ & $0(0.0 \%)$ & & & \\
\hline \multicolumn{7}{|c|}{ Inversions } \\
\hline 0 & $68(100.0 \%)$ & $36(97.3 \%)$ & $8(100.0 \%)$ & 0.352 & 1.000 & 0.398 \\
\hline 1 & $0(0.0 \%)$ & $1(2.7 \%)$ & $0(0.0 \%)$ & & & \\
\hline \multicolumn{7}{|c|}{ Monosomies } \\
\hline 0 & $62(91.2 \%)$ & $36(94.7 \%)$ & $6(75.0 \%)$ & 1.000 & 0.248 & 1.000 \\
\hline 1 & $4(5.9 \%)$ & $2(5.3 \%)$ & $1(12.5 \%)$ & & & \\
\hline 2 & $1(1.5 \%)$ & $0(0.0 \%)$ & $1(12.5 \%)$ & & & \\
\hline 5 & $1(1.5 \%)$ & $0(0.0 \%)$ & $0(0.0 \%)$ & & & \\
\hline \multicolumn{7}{|c|}{ Trisomies } \\
\hline 0 & $67(98.5 \%)$ & $37(97.4 \%)$ & $8(100.0 \%)$ & 1.000 & 1.000 & 1.000 \\
\hline 1 & $1(1.5 \%)$ & $1(2.6 \%)$ & $0(0.0 \%)$ & & & \\
\hline
\end{tabular}

$P$ value $<0.05$ was considered significant

Descriptive measures: $a( \pm b), a=$ average and $b=$ standard deviation 
Table 6 Statistical analysis of ATM/P1054R genotypes

\begin{tabular}{|c|c|c|c|c|c|}
\hline & \multicolumn{3}{|l|}{$A T M / P 1054 R$} & \multirow{2}{*}{$\begin{array}{l}P \text { value } \\
\mathrm{CC} \times \mathrm{GG}+\mathrm{CG}\end{array}$} & \multirow{2}{*}{$\begin{array}{l}P \text { value } \\
\mathrm{CC} \times \mathrm{CG}\end{array}$} \\
\hline & $\mathrm{CC}$ & CG & GG & & \\
\hline \multicolumn{6}{|l|}{ Gender $N(\%)$} \\
\hline Men & $42(47.2 \%)$ & $14(66.7 \%)$ & $1(100.0 \%)$ & 0.097 & 0.146 \\
\hline Women & $47(52.8 \%)$ & $7(33.3 \%)$ & $0(0.0 \%)$ & & \\
\hline \multicolumn{6}{|l|}{ Ethnicity $N(\%)$} \\
\hline Black & $25(27.8 \%)$ & $7(33.3 \%)$ & $0(0.0 \%)$ & 1.000 & 0.976 \\
\hline Brown & $47(52.2 \%)$ & $11(52.4 \%)$ & $1(100.0 \%)$ & & \\
\hline White & $14(15.6 \%)$ & $3(14.3 \%)$ & $0(0.0 \%)$ & & \\
\hline Asian & $2(2.2 \%)$ & $0(0.0 \%)$ & $0(0.0 \%)$ & & \\
\hline Native American & $2(2.2 \%)$ & $0(0.0 \%)$ & $0(0.0 \%)$ & & \\
\hline $\begin{array}{l}\text { Number of abnormal } \\
\text { metaphases per } \\
\text { subject }\end{array}$ & $0.74( \pm 1.36)$ & $0.62( \pm 1.12)$ & $1(N)$ & 0.924 & 0.913 \\
\hline $\begin{array}{l}\text { Number of chromo- } \\
\text { somal aberrations } \\
\text { per subject }\end{array}$ & $1.01( \pm 1.85)$ & $0.71( \pm 1.35)$ & $2(N)$ & 0.517 & 0.965 \\
\hline
\end{tabular}

Descriptive measures: $a( \pm b), a=$ average and $b=$ standard deviation populations were equivalent (Table 6). Only a weak positive association with chromosome breaks was detected, when compared between the genotypes with the variants $\mathrm{CC} \times \mathrm{GG}+\mathrm{CG}(P=0.054)$, as shown in Table 7 . To assess the capacity of variant alleles $R A D 51 / G 135 C$ and $A T M / P 1054 R$ to detect the workers with CAs, the sensitivity and specificity were calculated. Note a considerable specificity for $R A D 51 / G 135 C$ (87\%) and ATM/P1054R (79\%); however, lower sensitivity was found for both $28 \%$ and $14 \%$, respectively. When the specificity was calculated for $R A D 51 / G 135 C$ and $A T M / P 1054 R$ together, the value found was $82 \%$ (see Table 3 ).

\section{Discussion}

The association between two dysfunctional polymorphisms $R A D 51 / G 135 C$ and $A T M / P 1054 R$, and CAs, as an early effect biomarker, was evaluated in this cross-sectional study. Numerous studies have associated exposure to BTEX with increased levels of CAs in circulating lymphocytes of exposed workers (Zhang et al. 2002, 2012; Santiago et al. 2014; Gonçalves et al. 2016). Increased levels of CAs have, in turn, been correlated with an increased risk of cancer, especially for hematologic malignancies, such as myelodysplastic syndrome (MDS) and acute myelogenous leukemia (AML)—(Smith 2010).

FISH using wcp probes was applied in our study to detect alterations caused by chronic exposure to BTEX in $21.87 \%$ (chromosomes 1, 2, and 4, together) of the human genome (Verdorfer et al. 2001). Similar results were previously described by our research group (Santiago et al. 2014) applying the same technique in GSW populations (rate: 9.3 CAs per 1000 metaphases), as well as results described by Verdorfer et al (2001) in populations exposed to nitroaromates (16.0 CAs per 1000 metaphases) and compared to controls (5.85 CAs per 1000 metaphases). No CA was detected in the control group, fact that draws attention when compared to the high CAs frequency found in GSW group. It is possible GSWs with higher rates of CAs have a higher risk of developing cancer in future than others with low rates of CAs.

In the present study, the frequencies of $R A D 51 / G 135 C$ variant were higher in the GSW population when compared to controls, and the allele variant genotypes were associated with CAs per workers. In a meta-analysis study on the relationship between $R A D 51 / G 135 C$ and cancer risk, Zhao and cowokers (2014) investigated 42 studies involving 19,142 cases and 20,363 controls (Zhao et al. 2014). They found a significantly increased risk for overall cancers and concluded that $R A D 51 / G 135 C$ polymorphism is a candidate for susceptibility to cancer in general, especially for breast cancer. In another meta-analysis involving ten studies with, 656 patients and 3725 controls, the $R A D 51 / G 135 C$ polymorphism was associated with increased MDS risk, while no association was observed for acute leukemia (He et al. 2014). In our study, chromosome fragments and chromosome breaks were positively associated with variant allele genotypes. There is evidence that in Rad51 deficient cells stop in the G2/M phase and accumulate chromosomal breaks prior to cell death or unregulated cell growth, justifying the association found (Sonoda et al. 1998; Mishra et al. 2018).

Regarding the $A T M$ results, no differences were found in the proportion of carriers of the $A T M / P 1054 R$ variant between workers and controls. However, this proportion was considerably higher among our workers $(19.0 \%, 21$ out of 110) compared to prostate cancer patients $(9.5 \%, 25$ out 
Table 7 Statistical analysis of ATM/P1054R genotypes and chromosome alterations

\begin{tabular}{|c|c|c|c|c|c|}
\hline & \multicolumn{3}{|c|}{$A T M / P 1054 R$} & \multirow{2}{*}{$\begin{array}{l}P \text { value } \\
\mathrm{CC} \times \mathrm{CG}\end{array}$} & \multirow{2}{*}{$\begin{array}{l}P \text { value } \\
\mathrm{CC} \times \mathrm{GG}+\mathrm{CG}\end{array}$} \\
\hline & $\mathrm{CC}$ & CG & GG & & \\
\hline \multicolumn{6}{|c|}{ Translocations } \\
\hline 0 & $71(78.9 \%)$ & $16(76.2 \%)$ & $0(0.0 \%)$ & 0.771 & 0.781 \\
\hline 1 & $10(11.1 \%)$ & $4(19.0 \%)$ & $0(0.0 \%)$ & & \\
\hline 2 & $7(7.8 \%)$ & $1(4.8 \%)$ & $0(0.0 \%)$ & & \\
\hline 4 & $1(1.1 \%)$ & $0(0.0 \%)$ & $0(0.0 \%)$ & & \\
\hline 5 & $1(1.1 \%)$ & $0(0.0 \%)$ & $0(0.0 \%)$ & & \\
\hline \multicolumn{6}{|c|}{ Chr. Fragments } \\
\hline 0 & $78(87.6 \%)$ & $20(95.2 \%)$ & $0(0.0 \%)$ & 0.793 & 0.662 \\
\hline 1 & $9(10.1 \%)$ & $1(4.8 \%)$ & $0(0.0 \%)$ & & \\
\hline 2 & $1(1.1 \%)$ & $0(0 \%)$ & $0(0.0 \%)$ & & \\
\hline 3 & $1(1.1 \%)$ & $0(0 \%)$ & $0(0.0 \%)$ & & \\
\hline \multicolumn{6}{|c|}{ Chr. breaks } \\
\hline 0 & $84(93.3 \%)$ & $19(90.5 \%)$ & $0(0.0 \%)$ & 0.352 & 0.054 \\
\hline 1 & $5(5.6 \%)$ & $1(4.8 \%)$ & $0(0.0 \%)$ & & \\
\hline 2 & $0(0.0 \%)$ & $1(4.8 \%)$ & $2(100.0 \%)$ & & \\
\hline 3 & $1(1.1 \%)$ & $0(0.0 \%)$ & $0(0.0 \%)$ & & \\
\hline \multicolumn{6}{|c|}{ Deletions } \\
\hline 0 & $79(87.8 \%)$ & $20(95.2 \%)$ & $0(0.0 \%)$ & 0.275 & 0.279 \\
\hline 1 & $9(10.0 \%)$ & $0(0.0 \%)$ & $0(0.0 \%)$ & & \\
\hline 2 & $2(2.2 \%)$ & $1(4.8 \%)$ & $0(0.0 \%)$ & & \\
\hline \multicolumn{6}{|c|}{ Chr. derivatives } \\
\hline 0 & $87(96.7 \%)$ & $20(95.2 \%)$ & $0(0.0 \%)$ & 0.573 & 0.589 \\
\hline 1 & $1(1.1 \%)$ & $1(4.8 \%)$ & $0(0.0 \%)$ & & \\
\hline 2 & $2(2.2 \%)$ & $0(0.0 \%)$ & $0(0.0 \%)$ & & \\
\hline \multicolumn{6}{|c|}{ Inversions } \\
\hline 0 & $89(98.9 \%)$ & $21(100.0 \%)$ & $0(0.0 \%)$ & 1.000 & 1.000 \\
\hline 1 & $1(1.1 \%)$ & $0(2.7 \%)$ & $0(0.0 \%)$ & & \\
\hline \multicolumn{6}{|c|}{ Monosomies } \\
\hline 0 & $82(91.1 \%)$ & $19(90.5 \%)$ & $0(0.0 \%)$ & 0.799 & 0.808 \\
\hline 1 & $5(5.6 \%)$ & $2(9.5 \%)$ & $0(0.0 \%)$ & & \\
\hline 2 & $2(2.2 \%)$ & $0(0.0 \%)$ & $0(0.0 \%)$ & & \\
\hline 5 & $1(1.1 \%)$ & $0(0.0 \%)$ & $0(0.0 \%)$ & & \\
\hline \multicolumn{6}{|c|}{ Trisomies } \\
\hline 0 & $88(97.8 \%)$ & $21(100.0 \%)$ & $0(0.0 \%)$ & 1.000 & 1.000 \\
\hline 1 & $2(2.2 \%)$ & $0(0.0 \%)$ & $0(0.0 \%)$ & & \\
\hline
\end{tabular}

Descriptive measures: $a( \pm b), a=$ average and $b=$ standard deviation of 261) and controls (4.78\%, 22 out of 460) described by Meyer and coworkers (2007). A weak positive association between chromosome breaks and the variant ATM/P1054R was detected in our workers, suggesting that more studies are necessary for a final conclusion.

In the case of the $C H E K 2 / T 470 C$, no variant alleles were found in our study, possibly due to the low frequency in our study population. CHEK2/T470C is associated with reduced DNA repair ability and increased cancer susceptibility, such as breast cancer, colorectal cancer and prostate cancer (Han et al. 2013; Kilpivaara et al. 2006; Dong et al. 2003). In the USA, the CHEK2/T470C variant has been reported in
$1.2 \%$ of the population, while in Germany, the frequency was $2.2 \%$ in breast cancer cases and $0.6 \%$ in controls; and in Belarussian population, $5.7 \%$ in cases and $1.3 \%$ in controls (Bogdanova et al. 2005). It may be necessary to increase the number of workers to be analyzed to draw conclusions about the CHEK2/T470C polymorphism in the Brazilian GSWs.

The literature has also reported an influence of gene-gene interactions on cancer susceptibility. Several studies have shown that combinations of RAD51 and ATM variants may increase the risk for cancer development (Hallajian et al. 2017). In our study, no increase in specificity or sensitivity was found for the RAD51/G135C and ATM/P1054R 
polymorphism combinations for detecting CAs. Perhaps for an effective GSWs genomic instability monitoring and an increase in the sensitivity and specificity in detecting CAs, it will be necessary to evaluate not only more polymorphisms related to the DNA repair, but also polymorphisms related to BTEX detoxification (Kanuoriya et al. 2015; Fang et al. 2017).

\section{Risk behavior and prevention of cancer}

Hematological changes in classic blood tests were previously described (IARC 2018; Zhang et al. 2012; Silvestre et al. 2017). In the present study, a high rate of monocytes, eosinophils, basophils, and gamma-gt was found compared to controls, as previously described (Zhang et al. 2012; Mitri et al. 2015; Otero and Ornellas 2015). However, a lower rate of platelets and neutrophils was associated with the workers. Despite the higher rate of monocytes found in total of workers, a lower rate of monocytes was associated with a high number of CAs (group two), highlighting the importance of the simple classic blood test in monitoring their overall health. Recently, Getu et al (2020) studied GSW in Ethiopia. In disagreement to our study, they found that hematimetric values had a significant increment when compared with the control group. However, they considered that a larger sample size should be conducted to explore the impact of these chemicals on their population. So it will be useful to conduct a meta-analysis study to check points of agreement and disagreement in different world population.

We should also consider the high frequency of spontaneous abortions in the first trimester of pregnancy of total abortions reported by the female workers compared to female controls. This was also previously described by Silvestre and coworkers (2017) in a study with a lower number of female GSWs (Silvestre et al. 2017). Thus, the immediate absence of female workers to the gas station once pregnancy is confirmed is necessary to reduce the BTEX`s genotoxic and abortive effects. More maternal-child health studies are needed, since the female gender was associated with a higher number of CAs (group two). Women have shown faster benzene biotransformation than men, metabolizing 23-26\% more benzene, and its known that benzene must be bio-transformed to exert its toxic effects. Thus, women may be at greater risk, and environmental/biological limit values established in studies of male subjects may be inadequate (Brown et al. 1998; Angelini et al. 2012; Moro et al. 2017; Santiago et al. 2017).

\section{Conclusion}

Herein we describe a health survey and the consequent genome risks related to the chronic exposure to gasoline vapors as well as the possible ways to monitor such risks. CAs are standardized biomarkers used to identify not only the worker population at a higher risk of developing cancer, but also specific individuals who are susceptible to cancer development. The higher frequencies of the RAD51/G135C polymorphism in the GSW population and its association with higher CA frequency are a relevant result.

Increasing the knowledge of the DNA repair variations in maintaining the genomic stability and integrity in the GSWs is crucial for cancer prevention. As a result, a better understanding of inter-individual variations in susceptibility, with the identification of groups at higher risk, may provide a foundation for developing better prevention programs.

Acknowledgments GA is a PAPD Fellow, Programa de Apoio à Pesquisa of State University of Rio de Janeiro (UERJ), Brazil. RTS Qualitec/INOV Fellow of State University of Rio de Janeiro, (UERJ), Brazil. We thank the subjects who volunteered to participate in the study.

Author contributions $\mathrm{UBO}, \mathrm{GA}$ and $\mathrm{MHO}$ designed the study and applied for Research Ethics Board approval. FS, MMT and RTS recruited the workers. The cytogenetic data were analyzed by ABHA and FS. LRS analyzed all statistical data. MMRC contributed with facilities. FS prepared the manuscript draft with important intellectual input from TL, GA and MHO. All authors approved the final manuscript and had complete access to the study data.

Funding This publication was paid by Secretaria Estadual de Saúde do Rio de Janeiro (SES/RJ) \#080011026391.

Data availability The data used in the current study is available from the corresponding author on reasonable request.

\section{Compliance with ethical standards}

Conflict of interest The authors declare that they have no competing interest.

Ethics approval This study was approved by the research ethics committees of Hospital Universitário Pedro Erneto (HUPE), \#CAAE 34310014.9.0000.5259, and National Cancer Institute José Alencar Gomes da Silva (INCA), \#121/09. Both committees are located in Rio de Janeiro, Brazil.

Informed consent Written informed consents were obtained from all subjects for publication of this paper and any accompanying images. Registry and the registration no. of the study/trial: UERJ34310014.9.0000.5259/14; INCA-121/09.

Consent to participate Written informed consent for participation in this study was obtained according to the Brazilian law.

Consent for publication Written informed consent for publication of this study was obtained according to the Brazilian law. 
Open Access This article is licensed under a Creative Commons Attribution 4.0 International License, which permits use, sharing, adaptation, distribution and reproduction in any medium or format, as long as you give appropriate credit to the original author(s) and the source, provide a link to the Creative Commons licence, and indicate if changes were made. The images or other third party material in this article are included in the article's Creative Commons licence, unless indicated otherwise in a credit line to the material. If material is not included in the article's Creative Commons licence and your intended use is not permitted by statutory regulation or exceeds the permitted use, you will need to obtain permission directly from the copyright holder. To view a copy of this licence, visit http://creativecommons.org/licenses/by/4.0/.

\section{References}

Angelini S, Maffei F, Bermejo JL, Ravegnini G, L'insalata D, CantelliForti G, Violante FS, Hrelia P, (2012) Environmental exposure to benzene, micronucleus formation and polymorphisms in DNArepair genes: a pilot study. Mutat Res 743:99-104. https://doi.org/ 10.1016/j.mrgentox.2011.10.018

Bogdanova N, Enssen-Dubrowinskaja N, Feshchenko S, Lazjuk GI, Rogov YI, Dammann O, Bremer M, Karstens JH, Sohn C, Dörk $\mathrm{T}$ (2005) Association of two mutations in the CHEK2 gene with breast cancer. Int J Cancer 116:263-266. https://doi.org/10.1002/ ijc. 21022

Brown EA, Shelley ML, Fisher JW (1998) A pharmacokinetic study of occupational and environmental benzene exposure with regard to gender. Risk Anal 18:205-213. https://doi.org/10.1111/j.15396924.1998.tb00932.x

De Palma G, Manno M (2014) Metabolic polymorphisms and biomarkers of effect in the biomonitoring of occupational exposure to low-levels of benzene: state of the art. Toxicol Lett 231:194-204. https://doi.org/10.1016/j.toxlet.2014.10.007

Dewi R, Hamid ZA, Rajab NF, Shuib S, Razak A (2020) Genetic, epigenetic, and lineage-directed mechanisms in benzene-inducemalignancies and hematotoxicity targeting hematopoietic stem cells niche. Hum Exp Toxicol 39(5):577-595. https://doi.org/ $10.1177 / 0960327119895570$

Dong X, Wang L, Taniguchi K, Wang X, Cunningham JM, McDonnell SK, Qian C, Marks AF, Slager SL, Peterson BJ, Smith DI, Cheville JC, Blute ML, Jacobsen SJ, Schaid DJ, Tindall DJ, Thibodeau SN, Liu W (2003) Mutations in CHEK2 associated with prostate cancer risk. Am J Hum Genet 72:270-280. https:// doi.org/10.1086/346094

Falzone L, Marconi A, Loreto C, Franco S, Spandidos DA, Libra M (2016) Occupational exposure to carcinogens: Benzene, pesticides and fibers. Mol Med Rep 14(5):4467-4474. https://doi. org/10.3892/mmr.2016.5791

Fang Y, Wu H, Ye Y, Zhou L, Hu W, Zhang G, Sun P, Au W, Xia Z (2017) Association between polymorphisms of metabolic enzyme genes and chromosomal damage in benzene-exposed workers in China. J Occup Environ Med 59:215-e220. https:// doi.org/10.1097/JOM.0000000000001148

Getu S, Shiferaw E, Melku M (2020) Assessment of hematological parameters of petrol filling workers at petrol stations in Gondar town, Northwest Ethiopia: a comparative cross-sectional study. Environ Health Prev Med 25(1):44. https://doi.org/10.1186/ s12199-020-00886-1

Gonçalves RO, de Almeida MN, Rêgo MAV (2016) Association between occupational exposure to benzene and chromosomal alterations in lymphocytes of Brazilian petrochemical workers removed from exposure. Environ Monit Assess 188:334. https:// doi.org/10.1007/s10661-016-5340-1
Green MR, Sambrook J (2012) Molecular cloning. A laboratory manual, vol 1. Cold Spring Harbor Press Isolation and quantification of DNA, New York, pp 44-53

Hallajian Z, Mahjoubi F, Nafissi N (2017) Simultaneous ATM/ BRCA1/RAD51 expression variations associated with prognostic factors in Iranian sporadic breast cancer patients. Breast Cancer 24:624-634. https://doi.org/10.1007/s12282-016-0750-z

Han FF, Guo CL, Liu LH (2013) The effect of CHEK2 variant I157T on cancer susceptibility: evidence from a meta-analysis. DNA Cell Biol 32:329-335. https://doi.org/10.1089/dna.2013.1970

He YZ, Hu X, Chi X, Zhang Y, Deng X, Wei M, Wang Z, Zhou $\mathrm{Y}$ (2014) Association between RAD51 gene polymorphism $(-135 \mathrm{G} / \mathrm{C})$ and susceptibility of myelodysplastic syndrome and acute leukemia: evidence based on a meta-analysis. Tumor Biol 35:615-621. https://doi.org/10.1007/s13277-013-1085-4

IARC (2018) International Agency for Research on Cancer: Benzene IARC Monographs on the evaluation of carcinogenic risks to humans, vol 20. WHO Press, Geneva, pp 290-293

Kanuoriya YA, Kumar N, Gulati S, Aggarwal N, Gupta R (2015) Association of CYP2E1 and CYP1A1m2 (BsrD1) polymorphisms with cytogenetic biomarkers in petrol pump workers. Biomark Genomic Med 7:159-164. https://doi.org/10.1016/j. bgm.2015.11.003

Kilpivaara O, Alhopuro P, Vahteristo P, Aaltonen LA, Nevanlinna $\mathrm{H}$ (2006) CHEK2 I157T associates with familial and sporadic colorectal cancer. J Med Genet 43(7):e34. https://doi.org/10. 1136/jmg.2005.038331

Li K, Jing Y, Yang C, Liu S, Zhao Y, He X, Li F, Han J, Li G (2015) Increased leukemia-associated gene expression in benzeneexposed workers. Sci Rep 4:5369. https://doi.org/10.1038/srep0 5369

Liehr T, Claussen U (2002) FISH-Technology. Lab manual. Springer FISH on chromosome preparations of peripheral blood, Berlin, pp 73-81

Mateuca RA, Decordier I, Kirsch-Volders M (2012) Cytogenetic methods in human biomonitoring: principles and uses. Methods Mol Biol 817:305-334. https://doi.org/10.1007/978-1-61779-421-6_15

McHale CM, Zhang L (2012) Current understanding of the mechanism of benzene-induced leukemia in humans: implications for risk assessment. Carcinogenesis 33:240-252. https://doi.org/10.1093/ carcin/bgr297

Meyer A, Wilhelm B, Dörk T, Bremer M, Baumann R, Karstens JH, Machtens S (2007) ATM missense variant P1054R predisposes to prostate cancer. Radiother Oncol 83:283-288. https://doi.org/ 10.1016/j.radonc.2007.04.029

Mishra A, Saxena S, Kaushal A, Nagaraju G (2018) RAD51C/XRCC3 facilitates mitochondrial DNA replication and maintains integrity of the mitochondrial genome. Mol Cell Biol 38(3):e00489-e517. https://doi.org/10.1128/MCB.00489-17

Mitri S, Fonseca ASA, Otero UB, Tabalipa MM, Moreira JC, Sarcinelli PN (2015) Metabolic polymorphisms and clinical findings related to benzene poisoning detected in exposed Brazilian gas-station workers. Int J Environ Res Public Health 12:8434-8447. https:// doi.org/10.3390/ijerph120708434

Moro AM, Bruckera N, Charão MF, Baierle M, Sauer E, Goethel G, Barth A, Nascimento SN, Gauer B, Durgante J, Amaral BS, Neto FRA, Gioda A, Garcia SC (2017) Biomonitoring of gasoline station attendants exposed to benzene: effect of gender. Mutat Res 813:1-9. https://doi.org/10.1016/j.mrgentox.2016.11.002

Otero UB, Ornellas MH (2015) The health and environmental threats related to gas stations. In: Brown GA (ed) Health risk related to the exposition to benzene and other chemicals present in gasoline, 1st edn. Lambert Academic Publishing, Saarbrücken, pp 9-25

Rossner P, Boffetta P, Ceppi M, Bonassi S, Smerhovsky Z, Landa K, Juzova D, Srám RJ (2005) Chromosomal aberrations in 
lymphocytes of healthy subjects and risk of cancer. Environ Health Perspect 113(5):517-520. https://doi.org/10.1289/ehp. 6925

Santiago F, Alves G, Otero UB, Tabalipa MM, Scherrer LR, Kosyakova N, Ornellas MH, Liehr T (2014) Monitoring of gas station attendants exposure to benzene, toluene, xylene (BTX) using three-color chromosome painting. Mol Cytogenet 7:15. https://doi.org/10. 1186/1755-8166-7-15

Santiago F, Lima S, Pinheiro T, Silvestre RT, Otero UB, Tabalipa MM, Kosyakova N, Ornellas MH, Liehr T, Alves G (2017) Benzene poisoning, clinical and blood abnormalities in two Brazilian female gas station attendants: two case reports. BMC Res Notes 10:52. https://doi.org/10.1186/s13104-016-2369-8

Schumacher FR, Al Olama AA, Berndt SI et al (2018) Association analyses of more than 140,000 men identify 63 new prostate cancer susceptibility loci. Nat Genet 50(7):928-936. https://doi.org/ 10.1038/s41588-018-0142-8

Silvestre RT, Delmonico L, Bravo M, Santiago F, Scherrer LR, Moreira ADS, Tabalipa M, Otero U, Ornellas MHF, Alves G (2017) Health survey and assessment of the polymorphisms BRCA1/P871L, BRCA1/Q356R, and BRCA2/N372H in female gas station workers in Rio de Janeiro. Environ Mol Mutagen 58:730-734. https:// doi.org/10.1002/em.22120

Skasko E, Kluska A, Niwińska A, Kwiatkowska E, Bałabas A, Piątkowska M, Dąbrowska M, Nowakowska D, Pieńkowski T (2009) Age at onset of bilateral breast cancer, the presence of hereditary BRCA1, BRCA2, CHEK2 gene mutations and positive family history of cancer. Onkologie 32:182-188. https://doi.org/ $10.1159 / 000200930$

Smith MT. (2010) Advances in understanding benzene health effects and susceptibility. Annu Rev Public Health 31: 133-48. https:// www.annualreviews.org/doi/10.1146/annurev.publhealth.012809. 103646

Sonoda E, Sasaki MS, Buerstedde JM, Bezzubova O, Shinohara A, Ogawa H, Takata M, Yamaguchi-Iwai Y, Takeda S (1998) Rad51deficient vertebrate cells accumulate chromosomal breaks prior to cell death. EMBO J 17:598-608. https://doi.org/10.1093/emboj/ 17.2.598

Verdorfer I, Neubauer S, Letzel S, Angerer J, Arutyunyan R, Martus P, Wucherer M, Gebhart E (2001) Chromosome painting for cytogenetic monitoring of occupationally exposed and non-exposed groups of human individuals. Mutat Res 491(1):97-109. https:// doi.org/10.1016/S1383-5718(01)00128-0

Villalba-Campos M, Chuaire-Noack L, Sánchez-Corredor MC, Rondón-Lagos M (2016) High chromosomal instability in workers occupationally exposed to solvents and paint removers. Mol Cytogenet 9:46. https://doi.org/10.1186/s13039-016-0256-6

Zeng X, Zhang Y, Yang L, Xu H, Zhang T, An R, Zhu K (2018) Association between RAD51 $135 \mathrm{G} / \mathrm{C}$ polymorphism and risk of 3 common gynecological cancers: A meta-analysis. Medicine (Baltimore) 97(26):e11251. https://doi.org/10.1097/MD.0000000000 011251

Zhang L, Eastmond DA, Smith MT (2002) The nature of chromosomal aberrations detected in humans exposed to benzene. Crit Rev Toxicol 32:1-42. https://doi.org/10.1080/20024091064165

Zhang L, Lan Q, Ji Z, Li G, Shen M, Vermeulen R, Guo W, Hubbard AE, McHale CM, Rappaport SM, Hayes RB, Linet MS, Yin S, Smith MT, Rothman N (2012) Leukemia-related chromosomal loss detected in hematopoietic progenitor cells of benzeneexposed workers. Leukemia 26:2494-2498. https://doi.org/10. 1038/leu.2012.143

Zhao M, Chen P, Dong Y, Zhu X, Zhang X (2014) Relationship between Rad51 G135C and G172T variants and the susceptibility to cancer: a meta-analysis involving 54 case-control studies. PLoS ONE 9(1):e87259. https://doi.org/10.1371/journal.pone.0087259

Publisher's Note Springer Nature remains neutral with regard to jurisdictional claims in published maps and institutional affiliations. 